\title{
Das duale Masterstudium an der Dualen Hochschule Baden-Württemberg (DHBW)
}

\author{
Marcus Hoffmann und Ruth Melzer-Ridinger
}

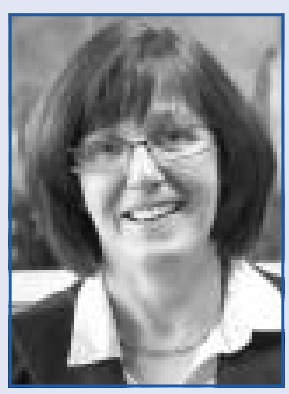

Prof. Dr. Ruth Melzer-Ridinger ist Dekanin der Fakultät Wirtschaft des Centers for Advanced Studies der DHBW in Heilbronn.

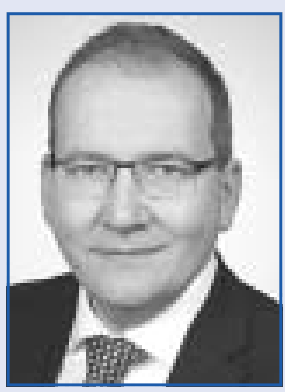

Prof. Dr. Marcus Hoffmann ist Prorektor für Studium und Lehre sowie Dekan der Fakultät Wirtschaft der DHBW Heidenheim.

Das duale Masterstudium des Centers for Advanced Studies der $D H B W$ bietet den Studierenden ein breitgefächertes Studienangebot in hoher Qualität. Das Studium an der $D H B W$ ermöglicht durch die vielfältigen und flexiblen Gestaltungsmöglichkeiten die Vereinbarkeit von Beruf, Weiterbildung und Privatleben.

Stichwörter: Berufsintegrierendes Masterstudium, berufsbegleitendes Masterstudium, Masterstudiengänge, Zulassungsvoraussetzungen, Bewerbungsverfahren

\section{Konzept und Organisation des dualen Masterstudiums an der DHBW}

Die $D H B W$ ist die erste duale, praxisintegrierende Hochschule in Deutschland. Gegründet am 1. März 2009 führt sie das seit 40 Jahren erfolgreiche duale Prinzip der früheren Berufsakademie Baden-Württemberg fort. An ihren neun Standorten und drei Campus bietet die $\mathrm{DHBW}$ in Kooperation mit ausgewählten Unternehmen und sozialen Einrichtungen eine Vielzahl von national und international akkreditierten Bachelor- und Masterstudiengängen in den Bereichen Wirtschaft, Technik und Sozialwesen an. Mit derzeit rund 34.000 Studierenden und über 141.000 Alum- ni ist die $D H B W$ die größte Hochschule in Baden-Württemberg.

Das Center for Advanced Studies (CAS) ist eine auf Masterprogramme und Weiterbildungsangebote in den Bereichen Wirtschaft, Technik und Sozialwesen spezialisierte Institution der Dualen Hochschule Baden-Württemberg $(D H B W)$. Diese bündelt dort ihre berufsintegrierenden und berufsbegleitenden Masterstudiengänge. Primäre Zielgruppen für die Masterstudiengänge sind Hochschulabsolventen mit mindestens einjähriger Berufserfahrung. Die Lehrveranstaltungen finden zentral am $C A S$ in Heilbronn sowie dezentral an den beteiligten Standorten der $D H B W$ statt.

Das Studienkonzept verknüpft anwendungsorientiert und forschungsbasiert Theorie und Praxis. Das Duale Masterstudium der $\mathrm{DHBW}$ ist inhaltlich berufsintegrierend konzipiert, Lehrveranstaltungen greifen regelmäßig Fragestellungen der Praxis auf und ein Drittel der Prüfungsleistungen werden mit Projekten aus dem beruflichen Umfeld der Studierenden erbracht. Das Studium ist berufsbegleitend organisiert - kurze Präsenzphasen und ein intensives begleitetes Selbststudium erlauben es den Studierenden ihre qualifizierte berufliche Tätigkeit auch weiterhin engagiert auszuüben. Für das weiterbildende duale $D H B W$-Masterstudium ist mindestens ein Jahr Berufserfahrung nach dem Bachelorabschluss erforderlich. In dieser Zeit können allerdings einzelne Module bereits weiterbildend absolviert werden, die nach Aufnahme des Masterstudiums Anrechnung finden.

Das Curriculum der einzelnen Studiengänge orientiert sich an den Berufsbildern. Der modulare Aufbau der Masterstudienangebote des $C A S$ ermöglicht jedoch gleichermaBen einen hohen Grad an Wahlfreiheit bei der Zusammenstellung individueller Studienpläne. Dadurch werden individuelle Vorkenntnisse, berufliche Entwicklungsperspektiven sowie der Kompetenzbedarf des Arbeitgebers berücksichtigt.

\section{Vorteile für Studierende und ausbildende Partnerunternehmen}

Das Masterstudium am Center for Advanced Studies der $D H B W$ bietet folgende Vorteile für Studierende:

- Berufsintegrierend und berufsbegleitend: Keine Unterbrechung der beruflichen Karriere, Vereinbarkeit von 
Beruf, Weiterbildung und Privatleben sowie finanzielle Unabhängigkeit

- Hohe Qualität der Lehre: Professoren der $D H B W$ sowie Experten aus Unternehmen, kleine Kursgrößen (max. 25 Personen), persönliche Betreuung

- Netzwerk: Zugang zu branchenspezifischen Netzwerken sowie Aufbau neuer Netzwerke durch Kontakte zu Kommilitonen und Dozenten

- Vier der insgesamt zwölf zu belegenden Module können bereits im Jahr vor dem Masterstudium im Kontaktstudium absolviert und später für das Studium anerkannt werden.

- Flexible Gestaltung des Studiums: Bedarfsorientierte Modulauswahl sowie Studienorganisation auf Berufstätigkeit abgestimmt (Studium in geblockten Einheiten unter Nutzung von Feier- und Brückentagen, die Lehrveranstaltungen finden jeweils monatlich im Rahmen zwei- bis dreitägiger Tagesseminare und jeweils einer einwöchigen Präsenzwoche pro Semester statt). Die Bildungszeitgesetze der Länder ermöglichen den Studierenden Tage der bezahlten Freistellung für das Studium. So ermöglicht das Bildungszeitgesetz des Landes Baden-Württemberg jährlich eine bis zu fünf Tage dauernde bezahlte Freistellung. Zudem werden Schutzzeiten (Mutterschutz, Elternzeit und Pflegezeit) anerkannt. Aus wichtigem Grund sind zudem Beurlaubungen bis zu zwei Semestern möglich.

Die kooperierenden Ausbildungsunternehmen unterstützen das Masterstudium am Center for Advanced Studies der $D H B W$ aus folgenden Gründen:
- Persönliche und berufliche Weiterqualifizierung ,on the job“: Bedarfsgerechte und gezielte Personalentwicklung zur Bindung leistungsstarker Mitarbeiter, Aneignung von Fach- und Führungskompetenzen durch die Studierenden

- Anwendungsbezug: Inhaltlich flexible Gestaltung des Studiums angepasst an den Bedarf und die Einsatzmöglichkeiten im Unternehmen

\section{Die Masterstudiengänge der Fakultät Wirtschaft des CAS}

Die steigenden Herausforderungen in der Unternehmenspraxis verlangen von jungen Führungskräften und Fachexperten eine immer umfangreichere ökonomische Kompetenz. Die Masterstudiengänge der $D H B W$ am Center for Advanced Studies (CAS) im Bereich Wirtschaft befähigen die Teilnehmer größere Zusammenhänge im Management ihrer Unternehmen und Branchen zu verstehen. Das Studienangebot umfasst den Master in Business Management, wo, neben der Vermittlung von Know-how aus dem General Management, viel Wert auf branchenspezifisches Fachwissen innerhalb einer Studienrichtung (Accounting, Controlling und Steuern, Dienstleistungen, Finance, General Business Management, Handel (neu ab 2016), International Business, Marketing, Medien und Marketing, Personal und Organisation oder Supply Chain Management, Logistik und Produktion) gelegt werden kann (vgl. $A b b$. 1). Das breite Modulangebot kann auch dazu genutzt werden, sich umfassend weiterzuentwickeln - je nachdem, wie es die eigene berufliche Situation erfordert.

\section{GENERAL MANAGEMENT}

Forschung und Informationsmanagement

Methoden der Wirtschafts- und

Sozialforschung

Aktuelle Themen im

Informationsmanagement

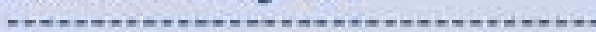

Ausgewahlte Managementthemen

zur Vertiefung (1 aus 2)

Qualitats- und Prozessmanagement oder

Innovations- und Change Management

Strategisches Management

Wertorientierte Unternehmensfuhrung

Ethikorientierte Unternehmensfuhrung

Rahmenbedingungen des Managements

Rechtliche Rahmenbedingungen

Managerial Economics

Organizational Behaviour and Leadership

Fuhrung von Mitarbeitern und Gruppen

Verhalten in und von Organisationen
Wisenschaftacties

Nbeten n der Praxis

STUDIENRICHTUNG

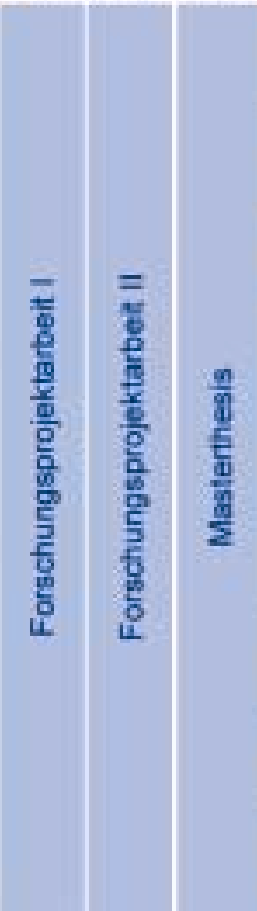

General Business Management

Accounting, Controlling und Steuem

Dienstleistungen

Finance

Internabonal Business

Marketing

Medien und Marketing

Personal und Organisation

Supply Chain Management. Logistik und Produktion

Abb. 1: Der Masterstudiengang Business Management im Überblick 
Im Master Steuern, Rechnungslegung und Prüfungswesen werden die Studierenden auf ihre umfassenden beruflichen Aufgaben vorbereitet. Zudem wird der Grundstein gelegt, um Berufsexamina in Steuerberatung und Wirtschaftsprüfung erfolgreich zu bestehen.

Führungskompetenz und Schlüsselqualifikationen sind weitere Inhalte des Masterstudiums. Denn dauerhafter Erfolg im komplexen, dynamischen und globalen Wirtschaftsgeschehen erfordert immer mehr die Kombination von fachlicher Exzellenz und integrativer Führungskompetenz. Die berufsintegrierenden Masterstudiengänge ermöglichen ein stark anwendungsorientiertes Studium. Themen aus dem konkreten Berufsalltag werden an der Hochschule besprochen und wissenschaftlich fundiert gelöst. Hierin entsteht ein direkter Mehrwert für das Unternehmen und für die Studierenden. Lerninhalte können viel besser vertieft werden, da sie eine direkte Anwendung in der Praxis finden.

Die Studierenden in der Fakultät Wirtschaft sollen befähigt werden, ihr erworbenes Wissen im beruflichen Alltag einzusetzen. Die Kompetenz, marktorientiert zu denken und zu handeln, wird dazu dienen, Probleme konzeptionell zu lösen oder die Ressourcen sowie die Strukturen eines Unternehmens zu managen.

Die Regelstudienzeit für das jeweils im Oktober startende Masterstudium beträgt 4 Semester (24 Monate). Verlängerungen sind möglich. Die Studiengebühren betragen 15.000 EUR (Stand: 1.2.2016). Während ihres Masterstudiums erwerben die Studierenden 90 ECTS. Als Abschluss erhalten die Absolventen der wirtschaftswissenschaftlichen Masterstudiengänge der $D H B W$ den Hochschulgrad „Master of Arts“ (M.A.) verliehen.

Die $D H B W$ wurde bereits 2011 als erste Hochschule in Baden-Württemberg von der $Z E v A$ systemakkreditiert. Somit werden auch die Masterstudiengänge des $C A S$ mit einem erstklassigen Qualitätsmanagement begleitet.

\section{Die Zulassungsvoraussetzungen für das Masterstudium}

Als Zulassungsvoraussetzung ist ein mindestens sechssemestriges Hochschulstudium mit wirtschaftswissenschaftlichem Schwerpunkt (z. B. BWL, Wirtschaftsinformatik oder Wirtschaftsingenieurwesen) erforderlich. Mit dem Studienabschluss müssen 210 ECTS-Punkte oder ein Diplomabschluss mit überdurchschnittlichem Ergebnis, d. h. ECTS-Klassifikation A oder B oder ein Notendurchschnitt von mindestens 2.5, erworben worden sein. Bewerber um einen Masterstudienplatz, die im Bachelor-Studium 180 ECTS-Punkte erworben haben, können zugelassen werden, wenn sie vor Beginn des Masterprogramms 30 ECTSPunkte in den vom CAS angebotenen Anpassungskursen erworben haben.

Zudem sind eine mindestens einjährige Berufstätigkeit nach Abschluss des Erststudiums sowie ein Beschäfti- gungsverhältnis mit einem betriebswirtschaftlichen Tätigkeitsschwerpunkt erforderlich.

Das Studium kann nur aufnehmen, wer an einem Beratungsgespräch teilgenommen hat, das

die Wissenschaftliche Leitung des Studiengangs oder eine von der Direktorin oder dem

Direktor des CAS beauftragten Person (Standortkoordinator/Standortkoordinatorin), die in der Regel eine Professorin oder ein Professor der Hochschule ist, durchgeführt hat. Das Zulassungsgespräch dient der Information und Beratung der Studienbewerberin oder des Studienbewerbers über Inhalte, Aufbau und Anforderungen eines Masterstudiums. Über die Teilnahme an dem Beratungsgespräch wird eine schriftliche Bescheinigung ausgestellt. In dem Beratungsgespräch werden die folgenden, für die spätere Zulassung erforderlichen Punkte geklärt:

- Liegt ein anzuerkennender Bachelorabschluss vor?

- Wenn kein Bachelorabschluss: Kann ein anderer Hochschulabschluss als gleichwertig anerkannt werden (wirtschaftswissenschaftliches Hochschulstudium oder Hochschulstudium mit wirtschaftswissenschaftlicher Fachrichtung mit mindestens 180 ECTS-Kreditpunkten, ein Studium an einer Berufsakademie nach badenwürttembergischen Modell oder ein rechtswissenschaftliches Hochschulstudium oder ein als gleichwertig anerkannten Auslandsstudium)?

- Wurden im Bachelorstudium grundlegende Kenntnisse der BWL erworben (gemäß $§ 13$ der Satzung über den Zugang und die Zulassung zu weiterbildenden Masterstudiengängen der $D H B W$ vom 13. März 2015)?

- Wenn ein fachfremdes Bachelorstudium abgeschlossen wurde: Wurden für das Studienprogramm notwendige Fachkenntnisse durch eine Prüfung nachgewiesen?

- Wurden mit dem Hochschulabschluss 210 ECTS erworben?

- Wenn keine 210 ECTS erworben wurden: Wurden Anpassungsmodule absolviert?

- Bachelorabschluss: ECTS-Klassifikation A oder B oder Gesamtnote mindestens 2,5?

- Liegt eine mindestens einjährige, fachlich einschlägige Berufserfahrung im Anschluss an den Erwerb eines Abschlusses gemäß $§ 3$ der Satzung über den Zugang und die Zulassung zu weiterbildenden Masterstudiengängen der $D H B W$ vom 13. März 2015 vor?

- Liegt eine Vereinbarung mit kooperierender Einrichtung gemäß $§ 3$ der Satzung über den Zugang und die Zulassung zu weiterbildenden Masterstudiengängen der $D H B W$ vom 13. März 2015 vor?

- Ist die kooperierende Einrichtung bereits als Dualer Partner zugelassen? 


\section{Das Kontaktstudium als Vorstufe des Masterstudiums}

Masterstudieninteressenten mit weniger als einem Jahr Berufspraxis können zur Überbrückung der Zeit bis zum Studienstart im sogenannten Kontaktstudium des CAS bereits erste Module des regulären Masterstudiums belegen und Prüfungsleistungen erbringen. Erfolgreiche absolvierte Module aus dem Kontaktstudium können für das spätere Masterstudium anerkannt werden.

Dabei können zum Beispiel im ersten Berufsjahr nach dem Bachelorabschluss praxisbegleitend bereits bis $\mathrm{zu} 20$ ECTS-Punkte (entspricht vier der zwölf insgesamt zu absolvierenden Module) aus dem Bereich General Management erworben werden. Hierfür ist keine Mastervereinbarung mit einem ausbildenden Partnerunternehmen erforderlich. Die Anmeldung für das Kontaktstudium, das jeweils am 1. Oktober eines Jahres beginnt, erfolgt direkt am CAS.

Durch die Teilnahme am Kontaktstudium lässt sich die spätere Belastung während des zweijährigen Masterstudiums verringern. Die Gebühren für die Belegung eines Moduls im Kontaktstudium betragen 1.200 EUR pro Modul (Stand 1.2.2016). Zusätzlich wird für die Teilnahme am Kontaktstudium eine Anmeldegebühr in Höhe von 120 EUR fällig. Alle für das Kontaktstudium angefallenen Modulgebühren werden später auf die Gebühren für das Masterstudium an der $D H B W$ angerechnet.

\section{Vorbereitung und Bewerbung für einen Masterstudienplatz an der DHBW}

Das CAS informiert Studieninteressierte auf vielfältige Weise. So werden an den $D H B W$-Studienakademien in Baden-Württemberg regelmäßig Informationsveranstaltungen zum dualen Masterstudium angeboten. Einen guten Überblick bietet auch das Informationsangebot des $C A S$ im Internet unter www.cas.dhbw.de. Zudem erhalten Bewerber/innen weitere Informationen des CAS über die aktuellen Entwicklungen des Masterstudienangebots, wenn sich diese einfach über das Internet unter URL: http://www.cas.dhbw.de/registrierung/registrieren lassen. An registrierte Studieninteressenten/innen versendet das $C A S$ aktuelle Benachrichtigungen zu Neuerungen sowie den Terminen der Masterinformationsveranstaltungen.

Um spezifische Fragen der Studieninteressenten im persönlichen Dialog zu klären bietet das $C A S$ diesen neben der allgemeinen Studienberatung Professoren als Ansprechpartner. Die Kontaktdaten der wissenschaftlichen Leiter sowie der Standortkoordinatoren des $C A S$ an den $D H B W$-Studienakademien können Studieninteressierte auf den studienrichtungsspezifischen Internetseiten des jeweiligen Masterangebots unter „Registrierung, Bewerbung und Kontakt" ersehen.

Vorschau auf WiSt Heft 12/2016

- Prof. Dr. Vanessa J. Haselhoff und Prof. Dr. Hartmut H. Holzmüller, Qualitative Marktforschung Teil 2: Datenerhebungsmethoden, Datenanalyse und Gütekriterien

- Oskar Gläser, M.Sc., Die steuerlichen Implikationen aus der Verlagerung von Wertschöpfungsaktivitäten ins Ausland - Die Besteuerung von grenzüberschreitenden Funktionsverlagerungen

- Prof. Dr. Hans Hirth und Martin Walther, M.Sc., Firmengröße im CAPM

- Prof. Dr. Michael Berlemann und Jascha Tutt, M.Sc., Banken und Finanzmärkte als Versicherung gegen Illiquidität - Eine numerische Darstellung des Diamond-Dybvig-Modells

- Prof. Dr. Michael Drewes, Hopfen und Malz, Gott erhalt's - Eine kleine Ökonomik des Bieres zum 500. Geburtstag des Reinheitsgebotes

- Prof. Dr. Tim Lohse und Oskar Jost, B.A., Zu erwartende Lohneffekte der Zuwanderung

- Benjamin Aust, MBA und Prof. Dr. Andreas Horsch, Das Wetterderivate-Paradoxon

- Prof. Dr. Daniela Lohaus und Prof. Dr. Uta Bronner, Interkulturelle Kompetenz

- Prof. Dr. Dirk Kaiser, Die Begebung einer Wandelanleihe - Teil l: Fallbeschreibung und Aufgaben 